\title{
Call for Papers: A Special Issue of Dreaming on the Arts
}

The journal Dreaming is planning a special issue on dreaming and the arts scheduled for publication in early 2003, to be guest-edited by Richard A. Russo. The deadline for submissions is March 1, 2002. Contributions are invited that (1) explore the role of dreams in particular works of art; (2) explore dreaming in relation to the arts in general, or to a specific artistic discipline; (3) explore how the study of dreaming can enhance our understanding of the arts and/or the creative process; or (4) explore how study of the arts can enhance our understanding of dreams. Although articles pertaining to literature will be considered, works that examine less-frequently explored arts, including painting, sculpture, film, theater, performance and dance, are particularly welcome.

Instructions to contributors are available on the back inside cover of Dreaming or at the ASD web site (www.ASDreams.org). Manuscripts will undergo full peer review and should be submitted by March 1, 2002, to: Richard A. Russo, M.A., 835 Peralta Ave., Berkeley, CA 94707 (RR@well.com). 